\title{
Superfluidity of pure spin current in ultracold Bose gases
}

\author{
Qizhong Zhu (朱起忠), ${ }^{1}$ Qing-feng Sun (孙庆丰), ${ }^{1,2}$ and Biao Wu (吴菻) ${ }^{1,2,3}$ \\ ${ }^{1}$ International Center for Quantum Materials, School of Physics, Peking University, Beijing 100871, China \\ ${ }^{2}$ Collaborative Innovation Center of Quantum Matter, Beijing 100871, China \\ ${ }^{3}$ Wilczek Quantum Center, College of Science, Zhejiang University of Technology, Hangzhou 310014, China
}

(Dated: October 13, 2018)

\begin{abstract}
We study the superfluidity of a pure spin current that is a spin current without mass current. We examine two types of pure spin currents, planar and circular, in spin-1 Bose gas. For the planar current, it is usually unstable, but can be stabilized by the quadratic Zeeman effect. The circular current can be generated with spin-orbit coupling. When the spin-orbit coupling strength is weak, we find that the circular pure spin current is the ground state of the system and thus a super-flow. We discuss the experimental schemes to realize and detect a pure spin current.
\end{abstract}

PACS numbers: 05.30.Jp, 03.75.Mn, 03.75.Kk, 71.70.Ej

\section{INTRODUCTION}

Since the experimental realization of Bose-Einstein condensation in optical traps, much effort has been devoted to the study of spinor superfluid [1 7]. With the degree of freedom of spin, spinor superfluid has much richer phases than scalar superfluid as it has both superfluid order and spin textures. However, most spinor superfluids studied till now carry both spin current and mass current. It will be interesting to see whether these two currents can decouple and further whether a pure spin current which has no mass current can flow frictionlessly. Our motivation also originates from condensed matter physics, in which the concept of spin superconductor [8, 9], formed by the Cooper-like pairs of electrons and holes and carrying spin super-current, is proposed. It is interesting to have these ideas realized in the field of cold atoms.

In this work we focus on the unpolarized spin-1 BoseEinstein condensate (BEC), where the pure spin current can be generated by applying a small magnetic gradient. It was found in Ref. [10] that a planar pure spin current in such a system is always unstable as the $m=1,-1$ components can collide into the $m=0$ component destroying the spin current. We find that the pure spin current can be stabilized with the quadratic Zeeman effect and become a super-flow. Furthermore, we propose a scheme to create pure spin current at the ground state, thus free from the issue of instability. Our scheme utilizes the spinorbit coupling. Specifically, we study a spin-1 BEC with Rashba spin-orbit coupling confined in a two-dimensional harmonic trap, and numerically find the ground state of the system. For antiferromagnetic interactions, opposite vortices appear in the $m=1,-1$ components with equal amplitude when the spin-orbit coupling is weak. Such a state carries pure spin current and no mass current. This spin current is a super-flow as it is the ground state and must be stable.

We note that there has been a lot of theoretical and experimental work on the counterflow of two species BEC [10 21]. For two miscible BECs with counterflow, it is found that there is a critical relative speed between the two species, beyond which the state is dynamically unstable [11 19]. It is shown that the instability can lead to proliferation of solitons [14, 15] and quantum turbulence 16]. This kind of counterflow is very similar to a spin current but it is not for two reasons: (1) Theoretically, if we regard the two species as two components of a pseudospin, this pseudo-spin has no $\mathrm{SU}(2)$ rotational symmetry as the number of bosons in each species is conserved. (2) Experimentally, it is hard to control the number of bosons in each component to create a spin current that has no mass current.

The paper is organized as follows. In Sec. III, we first study the stability of a spin-1 planar counterflow. We identify the mechanisms associated with the instabilities, and find that the quadratic Zeeman effect can stabilize such a planar flow. We then study the similar situation in the circular geometry in Sec. III. The pure spin current consists of a vortex and anti-vortex in the $m=-1,1$ components. The experimental schemes to realize the stable pure spin current is discussed in Sec. IV] Finally, we briefly summarize our main results in Sec. V.

\section{PLANAR FLOW}

The dynamics of a spin-1 BEC in free space is governed by the mean field Gross-Pitaevskii (GP) equation [7],

$$
i \hbar \frac{\partial}{\partial t} \psi_{m}=-\frac{\hbar^{2} \nabla^{2}}{2 M} \psi_{m}+c_{0} \rho \psi_{m}+c_{2} \sum_{n=-1}^{1} \mathbf{s} \cdot \mathbf{S}_{m n} \psi_{n}
$$

where $\psi_{m}(m=1,0,-1)$ are the components of the macroscopic wave function. $\rho=\sum_{m=-1}^{1}\left|\psi_{m}\right|^{2}$ is the total density, $\mathbf{s}_{i}=\sum_{m n} \psi_{m}^{*}\left(S_{i}\right)_{m n} \psi_{n}$ is the spin density vector and $\mathbf{S}=\left(S_{x}, S_{y}, S_{z}\right)$ is the spin operator vector with $S_{i}(i=x, y, z)$ being the three Pauli matrices in the spin-1 representation. The collisional interactions include a spin-independent part $c_{0}=4 \pi \hbar^{2}\left(a_{0}+2 a_{2}\right) / 3 M$ and a spin-dependent part $c_{2}=4 \pi \hbar^{2}\left(a_{2}-a_{0}\right) / 3 M$, with $a_{f}(f=0,2)$ being the $s$-wave scattering length for spin-1 atoms in the symmetric channel of total spin $f$. 
We consider a spin current state of the above GP equation with the form

$$
\psi=\sqrt{\frac{n}{2}}\left(\begin{array}{c}
e^{i \mathbf{k}_{1} \cdot \mathbf{r}} \\
0 \\
e^{i \mathbf{k}_{2} \cdot \mathbf{r}}
\end{array}\right),
$$

where $n$ is the density of the uniform BEC. The requirement of equal chemical potential leads to $\left|\mathbf{k}_{1}\right|=\left|\mathbf{k}_{2}\right|$. In the case where $\mathbf{k}_{1}=-\mathbf{k}_{2}$, this state carries a pure spin current: the total mass current is zero as it has equal mass counterflow while the spin current is nonzero.

It is instructive to first consider the special case when there is no counterflow, i.e., $\mathbf{k}_{1}=\mathbf{k}_{2}=0$. The excitation spectra are found to be $\epsilon^{0}=\sqrt{2 c_{2} n \epsilon_{q}+\epsilon_{q}^{2}}$ and $\epsilon_{1}^{ \pm 1}=\sqrt{2 c_{0} n \epsilon_{q}+\epsilon_{q}^{2}}, \epsilon_{2}^{ \pm 1}=\sqrt{2 c_{2} n \epsilon_{q}+\epsilon_{q}^{2}}$, respectively, with $\epsilon_{q}=\hbar^{2} q^{2} / 2 M$. So for antiferromagnetic interaction $\left(c_{0}>0, c_{2}>0\right)$, all branches of the spectra are real and there is a double degeneracy in one branch of the spectra. The phonon excitations give two sound velocities, $\sqrt{n c_{i} / M}(i=0,2)$, corresponding to the speeds of density wave and spin wave, respectively. However, the existence of phonon excitation does not mean that the pure spin current $\left(\mathbf{k}_{1}=\mathbf{k}_{2} \neq 0\right)$ is a super-flow as we can not obtain the current with $\mathbf{k}_{1}=\mathbf{k}_{2} \neq 0$ from the state with $\mathbf{k}_{1}=\mathbf{k}_{2}=0$ by a Galilean transformation.

The stability of the spin current has been studied in Ref. [10] for the case $\mathbf{k}_{1}=-\mathbf{k}_{2} \neq 0$. It is found that, for the antiferromagnetic interaction case $\left(c_{0}>0, c_{2}>0\right)$, the excitation spectrum of the $m=0$ component always has nonzero imaginary part in the long wavelength limit as long as there is counterflow between the two components, and the imaginary excitations in the $m=1,-1$ components only appear for a large enough relative velocity $v_{1}=2 \sqrt{n c_{2} / M}$. For the ferromagnetic interaction case $\left(c_{0}>0, c_{2}<0\right)$, both excitation spectra of the $m=0$ and $m=1,-1$ components have nonzero imaginary parts for any relative velocity. This means that the pure spin current cannot be stable in any cases.

For the general non-collinear case $\left(\mathbf{k}=\frac{\mathbf{k}_{1}+\mathbf{k}_{2}}{2} \neq 0\right)$ and antiferromagnetic interaction, the excitation spectrum for the $m=0$ component is found to be

$$
\epsilon^{0}=\sqrt{\left(\epsilon_{q}+\frac{\hbar^{2}}{2 M}\left(|\mathbf{k}|^{2}-\left|\mathbf{k}_{1}\right|^{2}\right)+c_{2} n\right)^{2}-c_{2}^{2} n^{2} .}
$$

We see here that as long as the momenta of the two components are not exactly parallel, i.e., $\mathbf{k}_{1}$ is not exactly equal to $\mathbf{k}_{2}$, then $|\mathbf{k}|<\left|\mathbf{k}_{1}\right|$, and there is always dynamical instability for the long wavelength excitations.

Therefore, the spin current in Eq. (2) is generally unstable and not a super-flow. This instability originates from the interaction process described by $\psi_{0}^{\dagger} \psi_{0}^{\dagger} \psi_{1} \psi_{-1}$ in the second quantized Hamiltonian. This energetically favored process converts two particles in the $m=1,-1$ components, respectively, into two stationary particles in the $m=0$ component. To suppress such a process and achieve a stable pure spin current, one can utilize the quadratic Zeeman effect. With the quadratic Zeeman effect of negative coefficient, the Hamiltonian adopts an additional term $\lambda m^{2}(\lambda<0$ and $m=1,0,-1)$. This term does not change the energy of the $m=0$ component, but lowers the energy of the other two components $m=1,-1$. As a result, there arises a barrier for two atoms in the $m=1,-1$ components scattering to the $m=0$ component, and the scattering process is thus suppressed.

The above intuitive argument can be made more rigorous and quantitative. Consider the case $\mathbf{k}_{1}=-\mathbf{k}_{2}$. With the quadratic Zeeman term, the excitation spectrum for the $m=0$ component changes to

$$
\epsilon^{0}=\sqrt{\left(\epsilon_{q}-\frac{\hbar^{2}\left|\mathbf{k}_{1}\right|^{2}}{2 M}+c_{2} n-\lambda\right)^{2}-c_{2}^{2} n^{2}} .
$$

So as long as $-\lambda-\hbar^{2}\left|\mathbf{k}_{1}\right|^{2} / 2 M>0$, long wavelength excitations will be stable for the $m=0$ component. From the excitation of the $m=0$ component, one can obtain a critical relative velocity of the spin current, $v_{0}=2 \sqrt{-2 \lambda / M}$. There is another nonzero critical velocity $v_{1}=2 \sqrt{n c_{2} / M}$ determined by the excitations of the $m=1,-1$ components. The overall critical velocity of the system is the smaller one of $v_{0}$ and $v_{1}$. Therefore, below the critical relative velocity $v_{\mathrm{c}}=\min \left\{v_{0}, v_{1}\right\}$, the pure spin current is stable and a super-flow. The experimental scheme to realize such a Zeeman effect will be discussed in Sec. IV]

\section{CIRCULAR FLOW}

In the cylindrical geometry, we consider a pure spin current formed by two vortices with opposite circulation in the $m=1,-1$ components. From similar arguments, one can expect that interaction will make such a current unstable. Inspired by the quadratic Zeeman effect method above, we propose to use spin-orbit coupling to stabilize it. The spin-orbit coupling can be viewed as a momentum-dependent effective magnetic field that exerts only on the $m=1,-1$ components. Therefore, it is possible that spin-orbit coupling lowers the energy of $m=1,-1$ components, and consequently suppresses the interaction process leading to the instability.

The model of spin-1 BEC subject to Rashba spin-orbit coupling can be described by the following energy functional,

$$
\begin{aligned}
\mathcal{E}\left[\psi_{\alpha}\right]= & \int d \mathbf{r}\left\{\sum_{\alpha} \frac{\hbar^{2}\left|\nabla \psi_{\alpha}\right|^{2}}{2 M}+\rho V(r)+\frac{c_{0}}{2} \rho^{2}+\frac{c_{2}}{2} \mathbf{s}^{2}\right. \\
& \left.+\gamma\left\langle S_{x} p_{y}-S_{y} p_{x}\right\rangle\right\}
\end{aligned}
$$

where $\rho$ is the density, $V(r)=\frac{1}{2} M \omega^{2}\left(x^{2}+y^{2}\right)$ is the trapping potential, and $\gamma$ is the strength of spin-orbit coupling. $\langle\cdots\rangle$ is the expectation value taken with respect to 
the three component wave function $\psi=\left(\psi_{1}, \psi_{0}, \psi_{-1}\right)^{T}$. The strength of the spin-orbit coupling $\gamma$ defines a characteristic length $a_{\text {soc }}=\hbar / M \gamma$, and can be rescaled to be dimensionless with respect to the harmonic oscillator length $a_{\mathrm{h}}=\sqrt{\hbar / M \omega}$. Then we characterize the strength of spin-orbit coupling with the dimensionless quantity $\kappa=a_{\mathrm{h}} / a_{\mathrm{soc}}=\gamma \sqrt{M / \hbar \omega}$. The spin-orbit coupling of Rashba type here can be generated in various ways, which will be discussed in the next section.

The above model can describe a spin- 1 BEC of ${ }^{23} \mathrm{Na}$ confined in a two-dimensional harmonic trap. Assume the atom number is about $10^{6}$. Using the estimate of scattering lengths $a_{0}=50 a_{B}, a_{2}=55 a_{B}$ [22], with $a_{B}$ being the Bohr radius, the ground state of spin- $1{ }^{23} \mathrm{Na}$ should be antiferromagnetic because $c_{0}>0, c_{2}>0[5]$. Previous studies of spin-1 BEC with Rashba spin-orbit coupling mostly focus on the strong spin-orbit coupling regime, where the ground state is found to be the plane wave phase or the stripe phase, for ferromagnetic interaction and antiferromagnetic interaction, respectively [23]. Here we are interested in the antiferromagnetic interaction case and the weak spin-orbit coupling regime $(\kappa \ll 1)$, and calculate the ground state wave function of the energy functional with the method of imaginary time evolution.

We find that when the spin-orbit coupling is weak $(\kappa \ll$ 1 ), the ground state wave function has the form

$$
\psi=\left(\begin{array}{c}
\chi_{1}(r) e^{-i \phi} \\
\chi_{0}(r) \\
\chi_{-1}(r) e^{i \phi}
\end{array}\right),
$$

with $\chi_{1}(r)=-\chi_{-1}(r)$ and all $\chi_{i}$ real. The ground state is shown in Fig. 1. Such a ground state consists of an anti-vortex in the $m=1$ component and a vortex in the $m=-1$ component. The $m=0$ component does not carry angular momentum. Since $\left|\psi_{1}\right|=\left|\psi_{-1}\right|$, the net mass current vanishes.

The wave function in Eq. (6) can be understood in the single particle level. In terms of the ladder operators of spin and angular momentum, the spin-orbit coupling term reads

$$
\mathcal{H}_{\mathrm{soc}}=\frac{\gamma \sqrt{M \hbar \omega}}{2}\left[S_{+}\left(\hat{a}_{R}-\hat{a}_{L}^{\dagger}\right)+S_{-}\left(\hat{a}_{R}^{\dagger}-\hat{a}_{L}\right)\right],
$$

where $S_{ \pm}$is the ladder operator of spin, and $\hat{a}_{L(R)}^{\dagger}$ is the creation operator of the left (right) circular quanta 24]. When the spin-orbit coupling is very weak $(\kappa \ll 1)$, its effect can be accounted for in a perturbative way. From the ground state $\Psi^{(0)}=|0,0\rangle$, the first order correction to the wave function for small $\gamma$ is given by

$$
\begin{aligned}
\Psi^{(1)} & =\frac{\gamma \sqrt{M \hbar \omega}}{2 \hbar \omega}\left(-S_{+} \hat{a}_{L}^{\dagger}+S_{-} \hat{a}_{R}^{\dagger}\right)|0,0\rangle \\
& =\frac{\kappa}{2}(-|1,-1\rangle+|-1,1\rangle),
\end{aligned}
$$

where $\left|m_{s}, m_{o}\right\rangle$ denotes a state with spin quantum number $m_{s}$ and orbital magnetic quantum number $m_{o}$. One (a1)

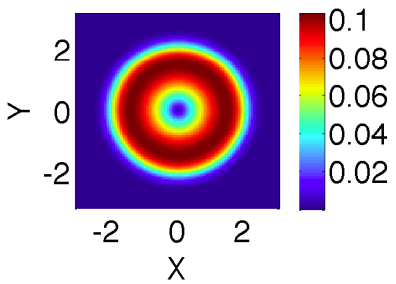

(b1)

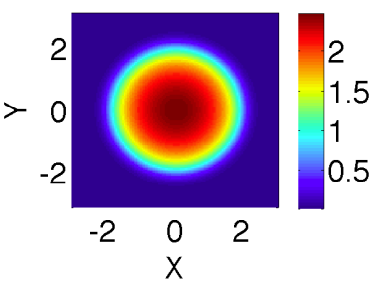

(c1)

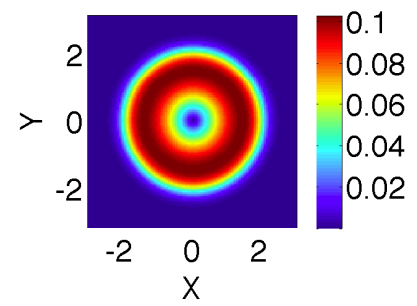

(a2)

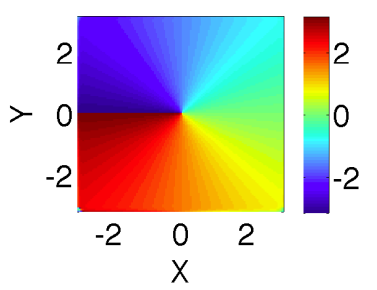

(b2)

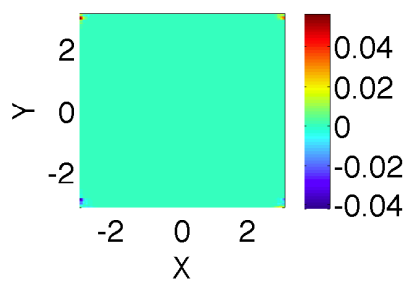

(c2)

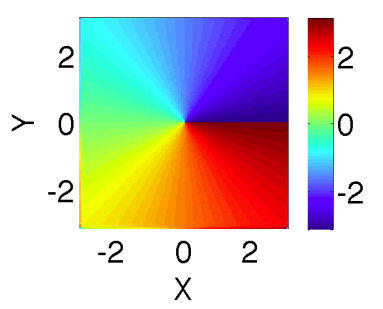

FIG. 1: (color online) Amplitudes (a1,b1,c1) and phase angles $(\mathrm{a} 2, \mathrm{~b} 2, \mathrm{c} 2)$ of the three component wave function $\psi=$ $\left(\psi_{1}, \psi_{0}, \psi_{-1}\right)^{T}$ at the ground state of Hamiltonian (5) for a $\mathrm{BEC}$ of ${ }^{23} \mathrm{Na}$ confined in a $2 \mathrm{D}$ harmonic trap. The particle number is $10^{6}$, the frequency of the trap is $2 \pi \times 42 \mathrm{~Hz}$, and the dimensionless spin-orbit coupling strength is $\kappa=0.04$. The units of the $X$ and $Y$ axes are $a_{\mathrm{h}}$.

immediately sees that $\psi_{1}$ has angular momentum $-\hbar$ and $\psi_{-1}$ has angular momentum $\hbar$. Besides, the amplitudes of both $\psi_{1}$ and $\psi_{-1}$ are proportional to $\kappa$.

There exits a continuity equation for spin density and spin current, which is

$$
\frac{d}{d t}\left(\psi^{\dagger} \mathbf{S}_{\mu} \psi\right)+\nabla \cdot \mathbf{J}_{\mu}^{s}=0 .
$$

The spin current density tensor $\mathbf{J}_{\mu}^{s}(\mu=x, y, z$ denotes the spin component) is defined as [25, 26]

$$
\begin{aligned}
\mathbf{J}_{\mu}^{s} & =\frac{1}{2}\left\{\psi^{\dagger} S_{\mu} \mathbf{v} \psi+\text { c.c. }\right\} \\
& =\frac{1}{2}\left\{\sum_{m, n, l} \psi_{m}^{*}\left(S_{\mu}\right)_{m n} \mathbf{v}_{n l} \psi_{l}+\text { c.c. }\right\},
\end{aligned}
$$

where

$$
\mathbf{v}_{n l}=\frac{\mathbf{p}}{M}+\gamma\left(\hat{z} \times \mathbf{S}_{n l}\right),
$$




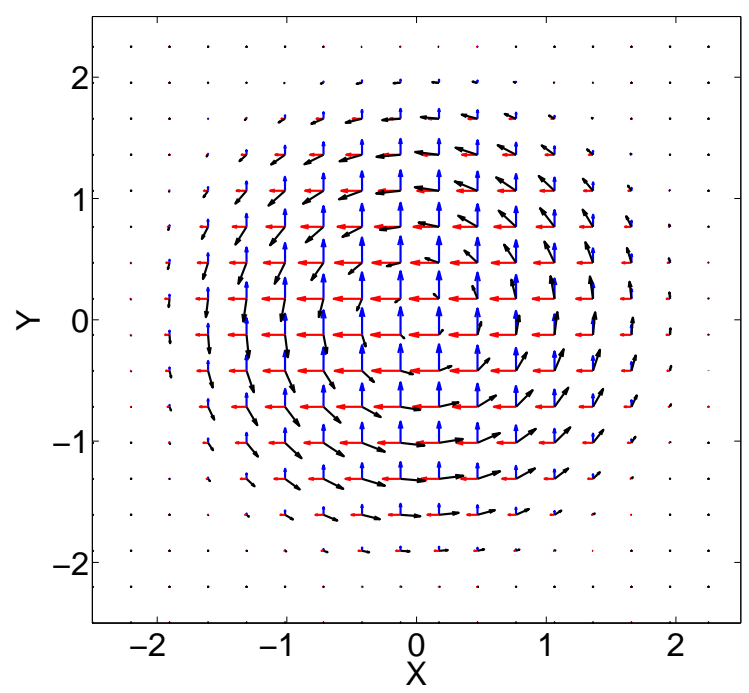

FIG. 2: (color online) Distribution of the spin current densities $\mathbf{J}_{x}^{s}$ (blue arrow), $\mathbf{J}_{y}^{s}$ (red arrow) and $\mathbf{J}_{z}^{s}$ (black arrow) of the ground state shown in Fig. 1. The length of the arrows represents the strength of the spin current. The arrow length of different colors is not to scale. $\kappa=0.04$. The units of the $X$ and $Y$ axes are $a_{\mathrm{h}}$.

and c.c. means the complex conjugate. The second part in $\mathbf{v}_{n l}$ is induced by the spin-orbit coupling.

By the definition in Eq. (10), the spin current density carried by the ground state (6) is

$$
\begin{aligned}
\mathbf{J}_{x}^{s}= & \gamma \sin 2 \phi\left|\psi_{1}\right|^{2} \hat{x}+\gamma\left(\left|\psi_{0}\right|^{2}+2\left|\psi_{1}\right|^{2} \sin ^{2} \phi\right) \hat{y} \\
\mathbf{J}_{y}^{s}= & -\gamma\left(\left|\psi_{0}\right|^{2}+2\left|\psi_{1}\right|^{2} \cos ^{2} \phi\right) \hat{x}-\gamma \sin 2 \phi\left|\psi_{1}\right|^{2} \hat{y} \\
\mathbf{J}_{z}^{s}= & \left(-\frac{2 \hbar\left|\psi_{1}\right|^{2}}{M r}+\sqrt{2} \gamma\left|\psi_{1} \psi_{0}\right|\right) \hat{\phi} .
\end{aligned}
$$

From both analytical and numerical results of the wave function, $\left|\psi_{1}\right| \ll\left|\psi_{0}\right|$, so $\mathbf{J}_{x}^{s}$ roughly points in the $y$ direction, while $\mathbf{J}_{y}^{s}$ almost points in the $-x$ direction. $\mathbf{J}_{z}^{s}$ represents a flow whose amplitude has rotational symmetry. From the numerical results shown in Fig. 2, we see that $\mathbf{J}_{z}^{s}$ is a counter-clockwise flow. The amplitudes of $\mathbf{J}_{x}^{s}$ and $\mathbf{J}_{y}^{s}$ are of the same order, both proportional to $\kappa$, while that of $\mathbf{J}_{z}^{s}$, proportional to $\kappa^{2}$, is much smaller. It is evident that the state in Eq. (6) carries no mass current and only pure spin current. Since the spin current is in the ground state, it must be stable. In this way, we have realized a superfluid of pure spin current, or a pure spin super-current.

\section{EXPERIMENTAL SCHEMES}

In this section, we propose the experimental schemes to generate and detect the pure spin currents discussed in Sec. II and Sec. III.
The planar pure spin current can be easily generated. By applying a magnetic field gradient, the two components $m=1,-1$ will be accelerated in opposite directions and a pure spin current is generated as done in Refs. [14, 15]. To stabilize this spin current, one needs to generate the quadratic Zeeman effect. We apply an oscillating magnetic field $B \sin \omega t$ with the frequency $\omega$ being much larger than the characteristic frequency of the condensate, e.g., the chemical potential $\mu$. The time averaging removes the linear Zeeman effect; only the quadratic Zeeman effect remains. The coefficient of the quadratic Zeeman effect from the second-order perturbation theory is given by $\lambda=\left(g \mu_{\mathrm{B}} B\right)^{2} / \Delta E_{\mathrm{hf}}$, where $g$ is the Landé $g$ factor of the atom, $\mu_{\mathrm{B}}$ is the Bohr magneton, and $\Delta E_{\mathrm{hf}}$ is the hyperfine energy splitting [27]. For the $F=2$ manifold of ${ }^{87} \mathrm{Rb}, \Delta E_{\mathrm{hf}}<0$, so the coefficient of the quadratic Zeeman effect is negative.

The circular flow in Sec. III may find prospective realizations in two different systems: cold atoms and exciton BEC. In cold atoms, we consider a system consisting of a $\mathrm{BEC}$ of ${ }^{23} \mathrm{Na}$ confined in a pancake trap, where the confinement in the $z$ direction is so tight that one can treat the system effectively as two dimensional. The spin-orbit coupling can be induced by two different methods. One is by the exertion of a strong external electric field $\mathbf{E}$ in the $z$ direction. Due to the relativistic effect, the magnetic moment of the atom will experience a weak spin-orbit coupling, where the strength $\gamma=g \mu_{B}|\mathbf{E}| / M c^{2}$. Here $M$ is the atomic mass and $c$ is the speed of light. For weak spin-orbit coupling (small $\gamma$ ), the fraction of atoms in the $m=1,-1$ components is proportional to $\gamma^{2}$. For an experimentally observable fraction of atoms, e.g., $0.1 \%$ of $10^{6}$ atoms, using the typical parameters of ${ }^{23} \mathrm{Na} \mathrm{BEC}$, the estimated electric field is of the same order of magnitude as the vacuum breakdown field. For atoms with smaller mass or larger magnetic moment, the required electric field can be lowered. Another method of realizing spin-orbit coupling is to exploit the atom laser interaction, where strong spin-orbit coupling can be created in principle 28]. In exciton BEC systems, as the effective mass of exciton is much smaller than that of atom, the required electric field is four to five orders of magnitude smaller, which is quite feasible in experiments 29 32 .

The vortex and anti-vortex in the $m=1,-1$ components can be detected by the method of time of flight. First one can split the three spin components with the Stern-Gerlach effect. The appearance of vortex or antivortex in the $m=1,-1$ components is signaled by a ring structure in the time of flight image. After a sufficiently long time of expansion, the ring structure should be clearly visible.

\section{CONCLUSION}

In summary, we have studied the stability of a pure spin current of a spin-1 BEC. In the planar flow, the system always suffers from dynamical instability. The 
origin of the instability is the interaction process that converts two particles in the $m=1,-1$ components into the $m=0$ component. Based on this, we propose a method to stabilize the pure spin current by utilizing the quadratic Zeeman effect. In the circular flow, we have proposed to use spin-orbit coupling to make the pure spin current stable. For weak spin-orbit coupling, we have found that the ground state of the system is a superfluid of pure spin current. The experimental schemes to realize and detect these pure spin currents have been discussed.

\section{Acknowledgements}

This work is supported by the NBRP of China (2013CB921903,2012CB921300) and the NSF of China (11274024,11274364,11334001,11429402).
[1] D. Hall, M. Matthews, J. Ensher, C. Wieman, and E. Cornell, Phys. Rev. Lett. 81, 4531 (1998).

[2] D. Hall, M. Matthews, C. Wieman, and E. Cornell, Phys. Rev. Lett. 81, 1543 (1998).

[3] M. Matthews et. al., Phys. Rev. Lett. 81, 243 (1998).

[4] J. Stenger et. al., Nature (London) 396, 345 (1998).

[5] T.-L. Ho, Phys. Rev. Lett. 81, 742 (1998).

[6] T. Ohmi, and K. Machida, J. Phys. Soc. Jpn. 67, 1822 (1998).

[7] See for example, D. M. Stamper-Kurn and M. Ueda, Rev. Mod. Phys. 85, 1191 (2013) and references therein.

[8] Q.-F. Sun, Z.-T. Jiang, Y. Yu, and X. C. Xie, Phys. Rev. B 84, 214501 (2011).

[9] Z.-Q. Bao, X. C. Xie, and Q.-F. Sun, Nat. Commun. 4, 2951 (2013).

[10] K. Fujimoto and M. Tsubota, Phys. Rev. A 85, 033642 (2012).

[11] C. K. Law, C. M. Chan, P. T. Leung, and M.-C. Chu, Phys. Rev. A 63, 063612 (2001).

[12] A. B. Kuklov and B. V. Svistunov, Phys. Rev. Lett. 90, 100401 (2003).

[13] V. I. Yukalov and E. P. Yukalova, Laser Phys. Lett. 1, 50 (2004).

[14] M. A. Hoefer, J. J. Chang, C. Hamner, and P. Engels, Phys. Rev. A 84, 041605(R) (2011).

[15] C. Hamner, J. J. Chang, P. Engels, and M. A. Hoefer, Phys. Rev. Lett. 106, 065302 (2011).

[16] H. Takeuchi, S. Ishino, and M. Tsubota, Phys. Rev. Lett. 105, 205301 (2010).
[17] S. Ishino, M. Tsubota, and H. Takeuchi, Phys. Rev. A 83, 063602 (2011).

[18] L. Y. Kravchenko and D. V. Fil, J. Low Temp. Phys. 155, 219 (2009).

[19] M. Abad, A. Sartori, S. Finazzi, and A. Recati, Phys. Rev. A 89, 053602 (2014).

[20] M. Vengalattore, S. R. Leslie, J. Guzman, and D. M. Stamper-Kurn, Phys. Rev. Lett. 100, 170403 (2008).

[21] R. W. Cherng, V. Gritsev, D. M. Stamper-Kurn, and E. Demler, Phys. Rev. Lett. 100, 180404 (2008).

[22] A. Crubellier et. al., Eur. Phys. J. D 6, 211 (1999).

[23] C. Wang, C. Gao, C.-M. Jian, and H. Zhai, Phys. Rev. Lett. 105, 160403 (2010).

[24] C. Cohen-Tannoudji, B. Diu, and F. Laloe, Quantum Mechanics, Vol. 1, Wiley, 1991.

[25] Q.-F. Sun and X. C. Xie, Phys. Rev. B 72245303 (2005).

[26] Q.-F. Sun, X. C. Xie, and J. Wang, Phys. Rev. B 77 035327 (2008).

[27] M. Ueda, Fundamentals and New Frontiers of BoseEinstein Condensation, World Scientific, 2010.

[28] J. Dalibard, F. Gerbier, G. Juzeliūnas, and P. Öhberg, Rev. Mod. Phys. 83, 1523 (2011).

[29] C. Weisbuch, M. Nishioka, A. Ishikawa, and Y. Arakawa, Phys. Rev. Lett. 69, 3314 (1992).

[30] J. Kasprzak et al., Nature 443, 409 (2006).

[31] K.G. Lagoudakis et al., Nat. Phys. 4, 706 (2008).

[32] A. Amo et al., Nat. Phys. 5, 805 (2009). 